\title{
INSINCERE UTTERANCES AND GAZE: EYE CONTACT DURING SARCASTIC STATEMENTS
}

\author{
Jason A. Williams: California Polytechnic State University, San Luis Obispo \\ Erin L. Burns: San Diego State University \\ Elizabeth A. Harmon: University of Washington
}

Summary:-Anecdotal evidence suggests that speakers often gaze away from their listeners during sarcastic utterances; however, this question has not been directly addressed empirically. This study systematically compared gaze-direction of speakers in dyadic conversation when uttering sincere and sarcastic statements. 18 naive participants were required to recite a series of contradictory statements on a single topic to a naive listener, while at the same time conveying their actual opinion about this topic. This latter task could only be accomplished through prosodic or nonverbal communication by indicating sincerity or insincerity (sarcasm) for the various statements and allowed examination of gaze across the two conditions for each participant. Subsequent analysis of the videotaped interaction indicated that, during the time for the actual utterance;' sarcastic utterances were accompanied by greater gaze aversion than were sincere utterances. This effect occurred for 15 of 18 participants ( 3 men, 15 women; M age $=19.8, S D=1.0$ ) who had volunteered for a small credit in an Introductory Psychology course. Results are discussed in terms of nonverbal communication and possible miscommunication which may apply given cultural differences in use of nonverbal cues.

Gaze direction, including eye contact, has been shown to be a powerful communicative tool in a variety of situations. It is affected by numerous social factors (e.g., Argyle \& Cook, 1976; Kleinke, 1986) and stimulates a number of personality attributions (e.g., Knackstedt \& Kleinke, 1991). One widespread belief shared by many western cultures is that eye contact indicates truthfulness in speech (Bond, Omar, Mahmoud, \& Bonser, 1990; Vrij \& Semin, 1996). The Global Deception Research Team (2006) reported 63.6\% of all tested individuals believed that people avert their gaze when lying, constituting the most commonly believed lie-detection cue in 51 of the 58 countries. While no such relation actually occurs for either adults (DePaul, Lindsay, Malone, Muhlenbruck, Charlton, \& Cooper, 2003) or children. (Talwar \& Lee, 2002), the pervasive acceptance of this behef raises some interesting possibilities regarding verbal sarcasm. During ironic utterances, a person linguistically speaks an untruth but intends the speech act as a whole to convey the opposite meaning (sarcasm adds a caustic dimension to the mix; Gibbs, 1986). One interesting possibility is that gaze aversion, often used as an indicator of lying by listeners, is actively co-opted by sarcastic speakers to cue listeners that some form of truth telling is not currently occurring in a communication of the true intended meaning: "I'm not looking at you, therefore my words are untrue." The current study directly addressed this issue.

People utter sarcastic comments on a daily basis to express hostile attitudes toward, or ridicule of, some other individual (Leggitt \& Gibbs, 2000). Most studies concerning sarcasm have examined phonological differences from normal speech, i.e., the prosody of verbal irony. Many types of sarcastic speech cues have been reported, although these vary 
by individual and circumstance and are likely mediated by how familiar are the conversational participants (Kreuz, Kassler, Coppenrath, \& McLain Allen, 1999; Rockwell, 2003). Some prosodic cues include slowed speech rate and nasalization (Cutler, 1974), monotonous intonation and long pauses between words (Haiman, 1998), and most consistently, stable pitch-contour (Shapely, 1987; Milosky \& Wrobleski, 1994; Haiman, 1998). Attardo, Eisterhold, Hay, and Poggi (2003) argued that there is no single ironic intonation, but rather prosodic cues are based on a departure from an individual's normal speech pattern. Their position is further supported by the subsequent study and analysis by Bryant and Fox Tree (2005).

In the interpretation of affect however, facial expressions have more influence on a listener than auditory information (DePaulo, Rosenthal, Eisenstat, Rogers, \& Finkelstein, 1978), and recent research supports the idea that there are specific visual markers of sarcastic utterances. Rockwell (2001) reported visual cues of sarcasm in the mouth region with naive participant speakers, and Attardo, et al. (2003) found numerous visual markers when examining American actors in situational comedies on television. These included raised or lowered eyebrows; open, squinting, or rolling eyes; winking; nodding; smiling; and no expression at all or what these authors called the "blank face."

Direction of gaze is a powerful cue under most social conditions which seems unexamined in this context. It was hypothesized that to convey sarcasm when there is little familiarity between people, individuals would avert their gaze to indicate the insincerity of the verbal component of the speech act. This issue was examined in pairs of participants. One member of a pair was designated to recite a series of contradictory statements, while at the same time communicating the true attitude to the listener. Conveying true beliefs required some cue outside the literal meaning of the specific utterances. While not true spontaneous sarcasm, this manipulation may provide more ecologically valid data than asking participants to be sarcastic (posed sarcasm). Only individuals with at most surface familiarity with each other were paired simply to reduce extraneous variation in the sample and to ensure sarcasm was necessary to complete the task. A video of the interaction was recorded on computer, and eye contact compared between the sincere and sarcastic utterances.

\section{Method}

\section{Participants}

Eighteen pairs of undergraduate university students ( 3 men, 15 women), who received a small credit in an Introductory Psychology class constituted the data set for the current experiment. All were born between 18 and 22 years of age $(M=19.9, S D=1.0)$ and were treated in accord with the policy of Gonzaga University Institutional Review Board. The data of a number of participants were not analyzed. Data from one pair were omitted after discovering during debriefing they were well-acquainted despite contrary instructions during sign-up. Data of eight participants were excluded from analysis because they moved outside the viewing area of the fixed camera during the orations. Data from eight other participants were omitted from analysis for lack of strong opinion to the topic, which invalidated the manipulation of sarcasm. Two others' data were excluded because their position to the listeners was not communicated. 


\section{Procedure}

To produce sarcastic utterances within a controlled laboratory setting, the following procedure was used. Participants signed up for the experiment in pairs, with the understanding that each should have had at most casual familiarity with the other person. Upon arriving, they were randomly designated as "speaker" and "listener," and escorted to a small ( $6 \mathrm{ft}$. X $10 \mathrm{ft}$.) well-lit room. The two participants sat in designated chairs facing each other about $4 \mathrm{ft}$. apart, and the speaker was given the following instructions, with the listener present: "You will be asked to read a series of 12 cards one at a time. On each is a statement about President Bush- six are positive, while six are negative. Your goal is to convey to the listener what your true feelings are toward the President. Please read each card, drop it in the bucked to your right, and then state the sentence exactly as written.

The listener was then instructed that the goal was simply to inter the speaker's true feelings toward the President. Each statement was designed to that it could equally be read sincerely or sarcastically, e.g., "Bush is a great President" and "Any Democrat would be better than Bush." Following the oration of the 12 statements, the participants were asked to rate on a 6-point scale his opinion of the President, anchored by 1: Strongly approve and 6: Strongly disapprove. The listener was given the same scale but asked to rate the speaker's opinion of the President.

\section{Video Recording and Analysis}

A Canon Optura Xi video camera was set up in the back of the room, aimed at the chair in which the speaker was to sit, and focused slightly wider than a full face shot when participants were present to allows some latitude for participant's height and posture (Fig 1). To make the speaker-listener interaction as natural as possible, no instructions regarding seating height, body posture, and so forth were given and no adjustments of the camera were made in their presence. While this resulted in many cases whom data were useless as the camera frame was misaligned from the actual head position, this disadvantage was offset by simply having participants behave without instructions regarding their motor behavior.

The spacial quality of the video recording (see below) allowed fairly easy assessment of gaze direction, since when the orator was looking at the other participants, they were almost making eye contact with the camera behind this persons' head. The situation was slightly complicated by the fact that the camera was minimally offset horizontally from the speaker-listener line, given occlusion for the listener's head. Therefore, three naïve coders were assigned blindly to assess the speaker's eye contact with the listener according to the following procedure.

First, data analysis was restricted to the interval of the actual utterance. Consistent with the literature (Levine \& Sutton-Smith, 1973), in all cases participants began their utterances while making eye contact. Since the process of reading the card, placing it in the bucket, and so forth represented behaviors of no theoretical interest, these were excluded, and while information may have been conveyed slightly before or after the utterance, no such signs were clearly visible. For each participant, the video session was edited into 12 segments, separated by a blank interval, with each segment consisting solely of the video which began at the frame when the utterance began and ended with the frame at which the utterance concluded. The audio was then removed, and 12 such segments were supplied to the three naive data coders to evaluate eye contact. 
Each coder advanced the video segment frame-by-frame and noted when eye contact was made or broken. The capture rate of $14 \mathrm{~Hz}$ fixed frames $70 \mathrm{msec}$. apart While this temporal resolution may seem coarse, the average saccade requires $50 \mathrm{msec}$. from beginning to end of the actual movement (Hyde, 1959), and so high spatial resolution (800 x 600 pixels) was valued over having multiple frames of intersaccadic movement. For each utterance, coders subsequently summed the total duration of eye contact throughout the utterance. This figure was divided by the overall utterance length to yield a percentage of eye contact for that particular trial. Each of the three coders generated a percentage for each of the 12 statements, and a final value for each trial calculated as the mean of the three estimates. Reliability between coders was high (Cronbach alpha=.97).

\section{RESULTS AND DISCUSSION}

For each speaker, it was first necessary to identify which set of six questions was sarcastic and which was sincere; this was accomplished by examining their rating score of presidential approval. Those who had positive ratings for the President had statements favorable to the President coded as sincere and negative questions coded as sarcastic, with the reverse procedure used for those who were negative toward the President. Overall, four participants were positive, while 14 were negative. For each participant, the estimates of the six sincere and six sarcastic utterances were averaged separately for a single mean percentage of eye contact for the sincere and sarcastic utterances. Across the 18 participants, mean eye contact occurred $63.9 \%$ ( $S D=18.3 \%$ ) of the time for sincere statements, and $52.7 \%$ ( $S D=21.3 \%$ ) of the time for sarcastic statements, a difference which was statistically significant ([17=3.5, two-tailed $p=.003)$. Effect size was large, over $80 \%$ of a standard deviation $(d=.83$, calculated by the ratio of mean difference to SD of the difference distribution, preserving within-group design characteristics). The effect was observed for 15 of 18 participants, including all four supporters of the President. Power analysis for the medium effect size of . 3 was $24.9 \%$, and for the measured effect size of . 83 was $98.9 \%$.

Consistent with the hypothesis, gaze aversion was consistently employed during sarcastic utterances. This cue differs from the "rolling of the eyes" reported by Attardo, et al. (2003); that effect, a vertical movement away from fixation, occurred for only four participants and then only in a few trials. Instead, the gaze aversion was most often accomplished with a simple horizontal saccade and sustained fixation away from the listener. The second most common expression of aversion was a simple saccade and fixation downward.

Similar to the findings of Attardo, et al. (2003) regarding phonological markers, eyecontact during sarcasm was dependent upon the normal gaze pattern of the speaker, i.e., a decrease from baseline gaze. Speakers varied widely in the overall percentage of eye contact, the lowest being $10.8 \%$ and the highest $88.4 \%$ (SD=20.0\%); however, even in these extreme cases, eye contact decreased during sarcasm, and this pattern across all participants was further reflected by a decrease in the measured standard deviation $(S D=13.7)$ of participants' within-differences scores. As the current study examines pairs of relative strangers, it is possible that increased familiarity with the listener would show a speaker decreasing either or both prosodic and nonverbal cues of sarcasm, including gaze aversion, consistent with familiarity (Kreuz, et al., 1999). 
An alternative to the idea that sarcastic speakers are actively employing a gazeaversion cue in communication is the possibility that this behavior is a result of basic cognitive processes associated with cognitive load. By the age of $8 \mathrm{yr}$., children tend to avert gaze when engaged in cognitively demanding activities (Doherty-Sneddon, Bruce, Bonner, Longbotham, \& Doyle, 2002). It is possible that the additional cognitive load associated with being sarcastic produces aversion in this circumstance. In the literature on deception, DePaulo, et at. (2003) were skeptical that this occurs when people are lying, simply because people are so practiced at this task that lying may only be slightly more difficult than truth telling. One might well be skeptical about this occurring in the present experiment for participants were reciting sentences.

Given that the gaze aversion reported in the current study may be a direct result of specific cultural beliefs about eye contact and truth telling, this cue may "employ only in these cultures in which this belief is common. Native American Navajo culture (Knapp, 1972), as well as Mexican-Americans and Japanese cultures (Sue \& Sue, 1977) assign different meanings to direct gaze (hostility and lack of respect), so sarcastic communication across cultures could be severely compromised by a misinterpretation of this nonverbal cue. Such is likely as sarcastic statements are in general perceived to be less sarcastic than intended (Rockwell, 1999, 2001, 2003). Also, given that sarcastic comments are often directed at a particular person, the end result might be especially inflammatory or damaging. Researchers could examine directly whether gaze aversion during sarcasm is coupled with cultural beliefs on truth telling and also whether cultural differences about this belief would produce sarcastic misunderstandings.

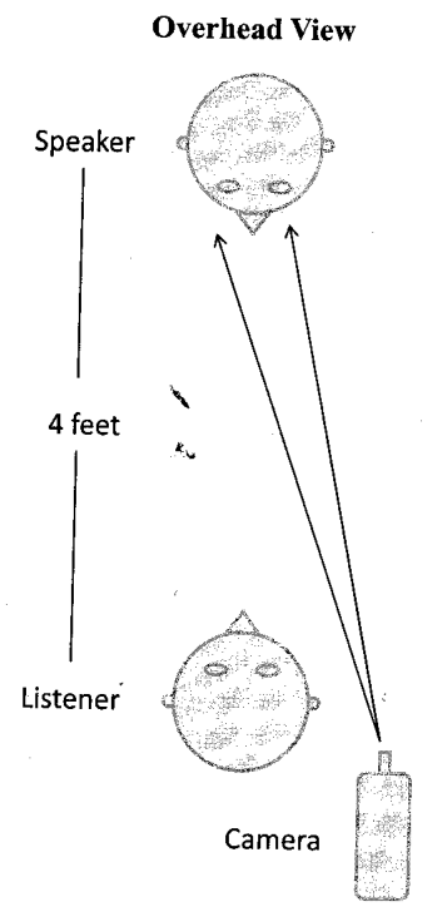

FIG. 1. Arrangement of speaker, listener, and camera 
TABLE 1

Percent of Eye Contact for Each of 18 Pairs of Participants

\begin{tabular}{cccc}
\hline Participant & Sincere & Sarcastic & Difference \\
\hline 1 & 65.5 & 49.8 & 15.7 \\
2 & 75.1 & 73.0 & 2.1 \\
3 & 68.4 & 66.3 & 2.1 \\
4 & 48.7 & 39.5 & 9.2 \\
5 & 66.3 & 43.4 & 22.9 \\
6 & 73.4 & 47.7 & 25.7 \\
7 & 74.0 & 57.6 & 16.4 \\
8 & 74.0 & 85.6 & -11.6 \\
9 & 59.3 & 69.3 & -10.0 \\
10 & 73.1 & 58.6 & 14.5 \\
11 & 69.7 & 23.6 & 46.1 \\
12 & 63.7 & 44.3 & 19.4 \\
13 & 47.7 & 30.0 & 17.7 \\
14 & 75.1 & 62.9 & 12.2 \\
15 & 94.2 & 85.6 & 8.6 \\
16 & 34.9 & 22.7 & 12.2 \\
17 & 75.1 & 79.4 & 4.3 \\
18 & 12.8 & 8.9 & 3.9 \\
\hline
\end{tabular}

\section{REFERENCES}

ARGYLE, M., \& COOK, M. (1976) Gaze and mutual gaze. Cambridge, MA: Cambridge Univer.Press.

ATTARDO, S., EISTERHOLD, J., HAY; J., \& POGGI, 1. (2003) Multimodal markers of irony and sarcasm. International Journal of Humor Research, 16, 243-260.

BOND, C. E, OMAR, A., MAHOUD, A., \& BONSER, R. N. (1990) Lie detection across cultures. Journal of Nonverbal Behavior, 14, 189-204.

BRYANT, G. A., \&Fox TREE, J. E. (2005) Is there an ironic tone of voice? Language and Speech, 48, 257 -277.

CUTLER, A. (1974) On saying what you mean without meaning what you say. In M. W. LaGaly, R. A. Fox, \& A. Bruck (Eds.), Papers from the Tenth Regional Meeting, Chicago Linguistic Society. Chicago, IL: Chicago Linguistic Society. Pp. 117-127. DEPAULO, B. M., LINDSAY, J. L., MALONE, B. E., MUHLENBRUCK, L., CHARLTON, K., \& COOPER, H. (2003) Cues to deception. Psychological Bulletin, 129,74-118.

DEPAULO, B. M., ROSENTHAL, R., EISENSTAT, R. H., ROGERS, P. L., \&FINKLESTEIN, S. (1978) Decoding discrepant nonverbal cues. Journal of Personality and Social Psychology, 36, 313-323.

DOHERTy-SNEDDON, G., BRUCE, V, BONNER, L., LONGBOTHAM, S., \&DOYLE, c. (2002) Development of gaze aversion as disengagement from visual location. Developmental Psychology, 38,438-445. 
GIBBS,GRe.neMra.l,(1191856,)3-105.n the psych Ii .. f 0 ngUlstlcs 0 sarcasm. Journal of Experimental Psychology:

GLOBAoLgyD,E3C7E,P6T0I-07N4.RESEARCH TEAM. (2006) A worId fl' 0 Ies. Journal of Cross-cultural Psychol-

HAIMAN, ]. (1998) Talk is cheap' l" UK: Oxford Univer. Press.. sarcasm, a zenatzon, and the evolution of language. Oxford,

HYDE, ]. E. (1959) Some characteristics of voluntarhItal plane. American Journal of Opthamology ocu ar movements 11 the horizon-

KLEINK1E0,0.C. L. (1986) Gaze and eye contact: a r"esearch.review. Psychological Bulletin, $100,78-$

KNACKTShTEDJT, G., I\&JKSLEINKE'"C L (1991) Eye contact gender and I" d e ourna OJ ocial Psychology, 131,303-304.' , persona lty JU gements.

KNAPPh, aMrt., 1\&. W(19in7s2t)on.Nonverbal commUlll'c"atlOn h III uman interaction. New York: Holt, Rine-

KREuz, R. ]., KASSLER, M. A. COPPENRATH L \&M LA common ground Tag questions and 1700. ve Irony. ourna OJ Pragmatics, 31, 1685-

LEGGIT2T9,, ]1. -S24,. \& GIBBS,R.W. (2000) Emotl.OnaI reactions to verbal irony. Discourse Processes

LEVINE, M. H., \& SUTTON-SMITH, B. (1973) Effects of a dUrIng dyadIC lllteraction

Developmental PhI g9' sex, and task on VIsual behavior

M L . syc oogy, , 400-405

ILOSKY, ., \&WROBLESKI, C. A. (1994) The Society for Humor Studies Confer IYho IroNny. Pyapekr presented at the Interna-

$\mathrm{R} p$ ( nce, t aca, ew or

OCKWELL, . 1999) Actors' partners' and Motor Skills, 89, 127-136. ' 0 servers perceptIOns

of sarcasm. Perceptual and

ROCKWELL, P (2001) Facial e p . d

ROCKWELL, P (2003) Em t::"- ressdlOnhan sarca.sm. Perceptual and Motor Skills, 93, 47-5 pa uy an $t$ e expressIOn and recogniti $f b$

tlons or strangers. Perceptual and Motor Skills 97 251-256 on 0 sarcasm y close rela-

SHAPEL6Y6-7M9: (1987) Prosod' .. d " . 1C varIatIOn an audience response. IprA: Papers in Pragmatics

UE, D. W, \&SUE, D. (1977) Barriers to ing Psychology, 24, 420-429. e ectIve cross-culture counseling. Journal of Counsel-

TALWAR, V, \& LEE, K. (2002) Development oftrol of expressive behavior during verbal ng $\mathrm{t}$ ? con1ceal a transgressIOn: children's convelopment, 26, 436-444. eceptlOn. nternatzonat Journal of Behavior De-

VRIJ, A., \& SEMIN, G. R.(1996) Lie expert's beliefs ab Journal of Nonverbal Behavior, 20, 6580 . 